\title{
Evaluation of mode of delivery in pregnant women infected with COVID-19
}

\author{
Paraskevi Giaxi ${ }^{1}$, Elissavet Maniatelli ${ }^{2}$, Victoria G. Vivilaki ${ }^{1}$
}

Childbearing women and newborn infants continue to require safe family-centered care during the current COVID-19 pandemic and they represent a vulnerable population ${ }^{1}$. In the only published systematic review of 108 pregnancies infected with COVID-19, 50 women were delivered, 44 gave birth by cesarean section and only 6 women gave birth by vaginal delivery ${ }^{2}$.

The World Health Organization (WHO) ${ }^{3}$, Royal College of Obstetricians \& Gynecologists and the Royal College of Midwives value equally each mode of delivery, however, opportunities should be provided to enable every woman to unlock their expectations and needs. Mode of birth should not be influenced by the presence of COVID-19, unless the woman's respiratory condition demands urgent intervention for birth ${ }^{3,4}$. In a symptomatic woman who is becoming exhausted or hypoxic, an individualized informed choice should be made regarding the possibility of shortening the length of the second stage of labor with elective instrumental birth ${ }^{4}$.

Caesarean section is not a recommended method of childbirth in pregnant women infected with COVID-19, however this was the mode of delivery in the majority of cases 5,6 with fetal distress cited as the indication behind the clinical decision. Moreover, 'fetal distress' is not justified by abnormal cardiotocograph findings or other factors like meconium stained amniotic fluid, abnormal foetal scalp lactate or blood flow changes. There was no evidence of COVID-19 in the amniotic fluid, umbilical cord blood, neonatal throat swab or breastmilk samples ${ }^{5}$. Vertical transmission was reported as negative in all neonates ${ }^{7}$.

Worldwide, CS rates are rising, and action needs to be taken, as was expressed in a recent Lancet series. CS rates in women with COVID-19 are even higher than in the general population. Protecting mothers from unnecessary medical technologies is one of the World Health Organization (WHO) strategies to promote maternal health. Under these circumstances, the role of midwife is more recognized as an advocate of natural birth for women ${ }^{8}$. Midwifery-led models of care are associated with lower cesarean utilization. The positive impact of midwife-led birth settings is well documented, including reductions in the need for a range of medical interventions ${ }^{9,10}$. These positive impacts remain of significant importance to prevent avoidable harm, and availability of midwife-led care settings for birth should therefore be continued as far as possible during the pandemic ${ }^{1}$.

The birth of COVID-19 pandemic is tokophobia. Frightened women are the products of this technocratic medicalized philosophy of birth. As 'obedient' consumers women are likely to choose intervention including Caesarean Section, distrusting the capacity of their own bodies to give birth normally. The influence of the media and significant technocratic believers, challenge the images of women and open the arena for a new understanding of safe birth during COVID-19 pandemic with Caesarean Section. Women need to be aware of their rights during this pandemic, that their own knowledge and feelings are valuable and, more importantly, that health research regarding COVID-19 is going on, however, Caesarean Section perinatal outcomes are well evident.

\section{REFERENGES}

1. The Royal College of Obstetricians and Gynecologists, Royal College of Midwives. Guidance for provision of midwife-led settings and home birth in the evolving coronavirus (COVID-19) pandemic: Information for healthcare professionals. https:// www.rcog.org.uk/globalassets/documents/guidelines/2020-05-22-guidance-forprovision-of-midwife-led-settings-and-home-birth-in-the-evolving-coronavirus-

\section{AFFILIATION}

1 Midwifery Department, University of West Attica, Athens, Greece

2 Second Department of Obstetrics and Gynecology, Aretaieion Hospital, National and Kapodistrian University of Athens, Athens, Greece

\section{CORRESPONDENCE TO}

Paraskevi Giaxi. Midwifery Department, University of West Attica, Athens, 12241, Greece. E-mail: voulagiaxi90@hotmail. com

\section{KEYWORDS}

COVID-19, pregnancy, mode of delivery, vaginal delivery section, midwifery-led models

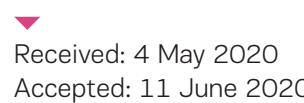


covid-19-pandemic.pdf. Published April 17, 2020. Accessed May 5, 2020.

2. Zaigham M, Andersson O. Maternal and Perinatal Outcomes with COVID-19: a systematic review of 108 pregnancies. Acta Obstet Gynecol Scand. 2020;00:1-7. doi:10.1111/aogs.13867

3. World Health Organization. Clinical management of severe acute respiratory infection (SARI) when COVID-19 disease is suspected: Interim guidance. https://apps.who.int/iris/bitstream/handle/10665/331446/WHO-2019-nCoVclinical-2020.4-eng.pdf?sequence=1\&isAllowed=y. Published March 13, 2020. Accessed May 5, 2020.

4. The Royal College of Obstetricians and Gynecologists, Royal College of Midwives. Coronavirus (COVID-19) infection in pregnancy: Information for healthcare professionals. https://www.rcog.org.uk/globalassets/documents/ guidelines/2020-06-04-coronavirus-covid-19-infection-in-pregnancy.pdf. Updated June 4, 2020. Accessed May 5, 2020.

5. Chen H, Guo J, Wang C, et al. Clinical characteristics and intrauterine vertical transmission potential of COVID-19 infection in nine pregnant women: a retrospective review of medical records. Lancet. 2020;395(10226):809-815. doi:10.1016/s0140-6736(20)30360-3

6. Liu H, Liu F, Li J, Zhang T, Wang D, Lan W. Clinical and CT Imaging Features of the COVID-19 Pneumonia: Focus on Pregnant Women and Children. J Infect. 2020;80(5):e7-e13. doi:10.1016/j.jinf.2020.03.007

7. Liu Y, Chen H, Tang K, Guo Y. Clinical manifestations and outcome of SARS-CoV-2 infection during pregnancy. $J$ Infect. 2020. doi:10.1016/j.jinf.2020.02.028

8. Hanahoe M. Midwifery-led care can lower caesarean section rates according to the Robson ten group classification system. Eur J Midwifery. 2020;4(March):1-5. doi:10.18332/ejm/119164

9. Scarf V, Rossiter C, Vedam S, et al. Maternal and perinatal outcomes by planned place of birth among women with low-risk pregnancies in high-income countries: A systematic review and meta-analysis. Midwifery. 2018;62:240-255. doi:10.1016/j.midw.2018.03.024

10. National Institute for Care Excellence. Clinical Guideline 190: Intrapartum care for healthy women and babies. nice.org. uk/guidance/cg190/chapter/recommendations\#place-of-birth. Updated February 21, 2017. Accessed April 8, 2020.

\section{CONFLICTS OF INTEREST}

The authors have completed and submitted the ICMJE Form for Disclosure of Potential Conflicts of Interest and none was reported.

\section{FUNDING}

There was no source of funding for this research.

\section{PROVENANCE AND PEER REVIEW}

Not commissioned; internally peer reviewed. 